\title{
Kurzübersicht der Studie
}

Die politische Interessenvermittlung durch Nichtregierungsorganisationen (NGOs) und andere zivilgesellschaftliche Akteure ist fester Bestandteil der Meinungsbildungs- und Entscheidungsprozesse in modernen GovernanceStrukturen. Zwischen der politischen Debatte und dem wissenschaftlichen Diskurs besteht jedoch eine Diskrepanz, mit welcher Intensität und mit welchen Themenschwerpunkten dieses Akteurs- und Handlungsfeld behandelt wird.

Die politische Debatte dreht sich um die Frage der übermäßigen Macht oder Ohnmacht von NGOs. Auf der einen Seite steht der wahrgenommene Bedeutungsgewinn, den NGOs als einflussreiche Akteure seit den 1990er Jahren in verschiedenen politischen Bereichen erlangt haben. Manche politischen Kreise kommen sogar zur Einschätzung, dass NGOs ihre Rolle als David gegen den Goliath der wirtschaftlichen Interessen und Unternehmen getauscht hätten. So belegten etwa die Mobilisierungserfolge bei den Demonstrationen gegen die Freihandelsabkommen TTIP/CETA sowie beim weltweiten Klimastreik der Fridays for Future-Bewegung, dass NGOs die „neuen Goliaths“ seien, die den politischen Diskurs in den Medien und der politischen Agenda dominierten. Auf der anderen Seite steht die Diskussion um die shrinking spaces der Zivilgesellschaft im Kontext des restriktiver werdenden Umgangs einiger europäischer und außereuropäischer Staaten mit NGOs. Eine solche Diskussion bildet sich inzwischen auch auf der nationalen Ebene ab. Sinnbildlich dafür sind das Attac-Urteil des Bundesfinanzhofs von Anfang 2019, welches der steuerlichen Gemeinnützigkeit enge Grenzen zog, der CDU-Parteitagsbeschluss Ende 2018, den Gemeinnützigkeitsstatus der Deutschen Umwelthilfe (DUH) aufgrund ihrer Rolle in der Feinstaubdiskussion rund um den Diesel-Skandal überprüfen zu wollen, oder die fortlaufende politische Debatte über eine Reform der Abgabenordnung im Gemeinnützigkeitsrecht in der ersten Jahreshälfte 2021. 
Die politik- und sozialwissenschaftliche Debatte hat das NGO-Thema aus verschiedenen Perspektiven gestreift und Teilaspekte beleuchtet. Trotz der Fortschritte des vergangenen Jahrzehnts bleibt der Wissensstand zu NGOs in der Interessenvermittlung beschränkt. Dies gilt insbesondere für das Spannungsfeld der Handlungslogiken von NGOs und anderen zivilgesellschaftlichen Organisationen im Feld der politischen Interessenvermittlung. Unklar bleibt, ob und wie sich die medial sichtbaren Erfolge von NGOs in substanziellem Politikwandel niederschlagen. Gleichzeitig rückt die gesteigerte politische Sichtbarkeit den Akteurstypus NGO an sich in das Interesse der kritischen Öffentlichkeit. Der politische Bedeutungszuwachs von NGOs korrespondiert insofern nicht mit einem politikwissenschaftlichen Erkenntnisgewinn gleichen Ausmaßes.

Die Studie „NGOs als besondere Akteure der Interessenvermittlung“, die vom Gemeinschaftsausschuss der Deutschen Gewerblichen Wirtschaft im Rahmen des Drittmittelprojekts „NGOs und Politikmanagement“ gefördert wurde, legt den Schwerpunkt auf NGOs als Interessenorganisationen im politischen Meinungsbildungs- und Entscheidungsprozess. Die Studie thematisiert die besondere Rolle von NGOs in der politischen Interessenvermittlung, die sich, so die Ausgangsthese, von anderen Interessengruppen unterscheidet. Ziel ist es, Erkenntnisse über die Handlungspraxis von NGOs im breiteren Kontext der modernen Interessenvermittlung zu gewinnen, weiterführende forschungsleitende Hypothesen zum Strategieportfolio von NGOs aufzustellen und zur konzeptionellen Weiterentwicklung des Wissensstandes zu Interessenvermittlungsorganisationen beizutragen.

Als Forschungspuzzle formuliert die Studie die Leitfrage: Wie agieren (verschiedene Typen von) NGOs im Rahmen von öffentlichkeitswirksamen Prozessen der Interessenvermittlung? Mit einem qualitativen Forschungsdesign vergleicht die Studie fünf NGOs - LobbyControl, Attac, Foodwatch, Campact und Deutsche Umweltstiftung (DUS) - die über verschiedene Strategie-Ausprägungen der Interessenvertretung (advocacy) sowie der Service- und Projektarbeit (non-advocacy) systematisch ausgewählt wurden. Daraus lassen sich zwar keine über alle NGOs hinweg generalisierbaren Aussagen treffen; die fünf Fälle decken allerdings eine umfängliche Bandbreite von Erscheinungsformen in der NGO-Welt ab. Diese fünf NGOs wurden als besonders aussagekräftige Fälle identifiziert und stehen stellvertretend im Fokus der Untersuchung. Als Datenmaterial dienen u. a. Experteninterviews und Dokumente, die mit Methoden der qualitativen Inhaltsanalyse ausgewertet werden (Abbildung 1.1). 


\section{Leitfrage}

„Wie agieren (verschiedene Typen von) NGOs in Prozessen der

Interessenvermittlung?“ Schwerpunkt auf spezifischer politischer Rationalität

\section{Empirischer Gegenstand}

Attac, LobbyControl, Foodwatch, Campact, Deutsche Umweltstiftung

fünf NGOs - möglichst verschieden, besonders typisch

\section{Theorie}

Dreieck der Handlungsrationalität für NGO-Strategien (advocacy/non-advocacy)

in institutionellem und politischem Kontext

\section{Methodik}

Qualitative Analyse auf Basis qualitativer Inhaltsanalyse

u.a. von Experteninterviews und Dokumenten

(Grafik: eigene Darstellung)

Abbildung 1.1 Die Eckpunkte der Studie auf einen Blick

Die Ergebnisse zeigen ein differenziertes Bild der verschiedenen Profilausprägungen aus einem umfassenden Strategieportfolio moderner Interessenvermittlung. Die modifizierte theoretische Perspektive eines Dreiecks der Handlungsrationalität gegenüber der Basis, der Politik und den Medien verdeutlicht die NGO-spezifischen Besonderheiten insbesondere in den Bereichen der übergeordneten Strategie, der Themenwahl, der Rolle von Expertise und der Legitimationsargumentation. Zusammengenommen weisen die Profilausprägungen auf den Mehrwert einer Binnendifferenzierung von public interest Organisationen hin, die die analytische Gegenüberstellung von gesellschaftlichen und wirtschaftlichen Interessen (business interests und public interests) verfeinert: In einer engen Begriffsbestimmung können NGOs als diffus-unterstützungsbasierter Subtyp nach dem Verbandsvorbild, als expertisebasierter Subtyp, als kampagnenorientierter Subtyp oder als plattformbasierter Subtyp gefasst werden. Mit dieser theoriegeleiteten und empirisch-explorativen Differenzierungsperspektive trägt die Studie dazu bei, das Verständnis von Strukturen und Prozessen des Politikmanagements von NGO-Strategien der Interessenvertretung (advocacy) sowie der Service- und Projektarbeit (non-advocacy) zu verbessern.

Das Buch beginnt mit dem spezifischen Erkenntnisinteresse der Studie und dem hierfür relevanten Forschungsstand (Kapitel 2), an den die Überlegungen zum Forschungsdesign anschließen (Kapitel 3). Im empirischen Teil steht zunächst 
eine Übersicht der Profile der fünf NGOs (Kapitel 4). Die darauf aufbauende qualitative Analyse stellt die verschiedenen Ausprägungen der Organisationen in den theoriegeleiteten und in den materialbasierten Untersuchungskategorien dar (Kapitel 5). Zum Abschluss entwirft die Studie praxisrelevante Bausteine moderner Interessenvermittlung (Kapitel 6) und formuliert abschließend Erkenntnisse in Form weiterführender Hypothesen (Kapitel 7). Hier können zukünftige Forschungsanstrengungen und die Generierung zusätzlicher Daten zum Gegenstand ansetzen.

Open Access Dieses Kapitel wird unter der Creative Commons Namensnennung 4.0 International Lizenz (http://creativecommons.org/licenses/by/4.0/deed.de) veröffentlicht, welche die Nutzung, Vervielfältigung, Bearbeitung, Verbreitung und Wiedergabe in jeglichem Medium und Format erlaubt, sofern Sie den/die ursprünglichen Autor(en) und die Quelle ordnungsgemäß nennen, einen Link zur Creative Commons Lizenz beifügen und angeben, ob Änderungen vorgenommen wurden.

Die in diesem Kapitel enthaltenen Bilder und sonstiges Drittmaterial unterliegen ebenfalls der genannten Creative Commons Lizenz, sofern sich aus der Abbildungslegende nichts anderes ergibt. Sofern das betreffende Material nicht unter der genannten Creative Commons Lizenz steht und die betreffende Handlung nicht nach gesetzlichen Vorschriften erlaubt ist, ist für die oben aufgeführten Weiterverwendungen des Materials die Einwilligung des jeweiligen Rechteinhabers einzuholen. 